\title{
A Simulation Comparison of Bootstrap Procedures in Periodically Correlated Time Series Models
}

\author{
Lorena Margo ${ }^{1}$, Eljona Milo ${ }^{2}$ \\ ${ }^{1}$ Department of Mathematics, Informatics and Physics, University "Fan S. Noli", Shëtitorja "Rilindasit", Korçë, \\ Albania \\ lorena.margo@yahoo.com \\ 2Department of Mathematics, Informatics and Physics, University “Fan S. Noli”, Shëtitorja "Rilindasit”, Korçë, \\ Albania \\ eljonamilo@yahoo.com
}

\begin{abstract}
The presence of periodicity in data with periodic structure has become an important issue in parameter estimation. Several methods have been studied with intention estimating different parameters or constructing confidence intervals for the parameters. In this paper we investigate the performance of the bootstrap procedures designed for dependent data in the case of Periodically Correlated time series models. Several models with periodic structure are studied in this paper and we use $\mathrm{R}$ programming language to realize a simulation comparison of the performance of bootstrap procedures presented.
\end{abstract}

\section{Keywords}

Block bootstrap; periodically correlated; time series.

\section{Academic Discipline And Sub-Disciplines}

Statistics

\section{SUBJECT CLASSIFICATION}

Statistical Subject Classification

\section{TYPE (METHOD/APPROACH)}

Application of Bootstrap procedures in estimating the seasonal means and the overall mean of several Periodically Correlated Time Series models. The R programming language is used to construct the programs for the algorithms used in the bootstrap procedures.

\section{INTRODUCTION}

The study of time series that contain a seasonal component has became an important issue nowadays. Periodically Correlated time series with period $T$ is a time series where the mean and covariance remains the same when shifted $T$ units of time. Since the introduction by Efron (1979), bootstrap was resulted to be an important method for estimating the distribution of an estimator by applying the resampling of the data. This method resulted efficient in the case of independent and identically distributed observations, but in the case of dependent data like time series, classical bootstrap gives incorrect answers. With intention to overcome this limitation block bootstrap is used, a modification of classic bootstrap that consists in resampling randomly with replacement the blocks compounded by a fixed number of observations. When the time series has a seasonal component we must take in consideration of the periodicity present in the data. The main purpose of this paper is to realize a simulation comparison of the performance of several bootstrap procedures in the case of estimating parameters in Periodically Correlated time series models. We use $R$ language in constructing the programs based in algorithms proposed in studying periodically correlated time series applying three bootstrap methods: Moving Block Bootstrap, Generalized Seasonal Block Bootstrap and Block Bootstrap of the Residuals. We will compare the results using several models of periodically correlated time series for different block lengths.

\section{METHODS AND RESULTS}

Periodic time series are encountered in different fields and the study of this special class of time series was resulted a challenge for many researchers. Periodically Correlated time series is a class of time series that is used to model data with periodic structure in many fields like climatology, meteorology, economy etc. A time series $x_{t}$ is called Periodically Correlated with period $T$ if the mean and the covariance of the series remains the same when shifted $T$ units of time. If we denote $\mathrm{E}$ the mean and cov the covariance, we have that $X_{t}$ is periodically correlated with period $\mathrm{T}$ if:

$E\left(X_{t+T}\right)=E\left(X_{t}\right), \operatorname{cov}\left(X_{t+T}, X_{s+T}\right)=\operatorname{cov}\left(X_{t}, X_{s}\right)$ for all integers s and t.

Moving block bootstrap (MBB) was proposed by Kunsch (1989) and Liu and Singh (1992). This procedure resamples blocks of consecutive observations at a time and as a result the dependence structure is preserved within each block. We will use the circular version of Moving Block Bootstrap. Using the circuar version we ensure that each of original observations receives equal weight so this is a property that distinguish this version from the non-circular version of MBB. Generalized Seasonal Block Bootstrap (GSBB) is a bootstrap method proposed by Dudek et. al. (2014) for periodically 
correlated time series. This method resulted to be suitable for periodic time series with fixed length periodicities of arbitrary size as related to block size and sample size. Another block bootstrap method for periodic time series, that resulted a real competitor of other block bootstrap procedures, is the Block Rootstrap resampling of the 'Residuals' (RBB). It consists in using block bootstrap resampling in the residuals of the original series after the seasonal means are estimated and removed. In some models of Periodically Correlated time series this block bootstrap procedure empirically gives good results compared to the other methods.

We perform a simulation study to calculate the mean squared error (MSE) and the bias of the estimators for the seasonal means and the overall mean using the algorithms of the proposed bootstrap procedures in the models proposed of periodically correlated time series. The $\mathrm{R}$ programming language is used to write the programs for the proposed bootstrap algorithms. We are focused in these time series models with periodic structure:

Model 1: $X_{t}=\sin (2 \pi t / T)+e_{t} \cos (2 \pi t / T)$; Model 2: $X_{t}=\cos (2 \pi t / T)+u_{t} \sin (2 \pi t / T)$ and

Model 3: $X_{t}=e_{t} \cos (2 \pi t / T)+u_{t} \sin (2 \pi t / T)+\cos (2 \pi t / T)$, where $u_{t}$ are independent random variables uniformly distributed on $[-0.5,0.5]$ and $e_{t}$ are independent random variables with standard normal distribution.

These series although do not have properly periodic covariance, they have a properly periodic mean, so it is important the study of these time series models. We generate time seres of length $N=120$ with number of bootstrap replications $B=500$ and we also perform 500 Monte-Carlo trials. We choose the period length $T=12$, and the block lengths are from the set $\{5,6,7,10,12,15,20,25,30\}$. The results are presented in the figures below:
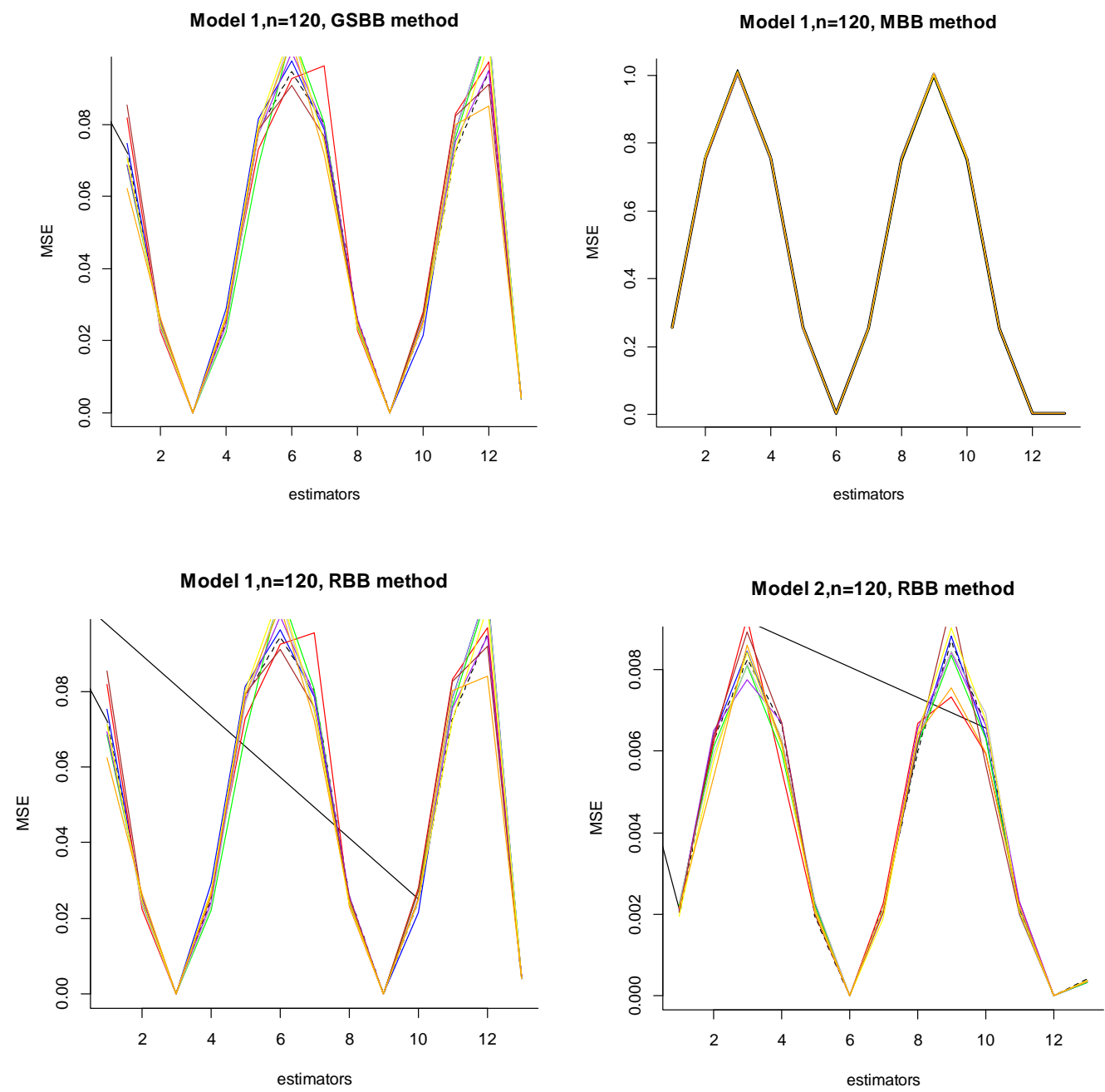

$6641 \mid \mathrm{P}$ a g e 

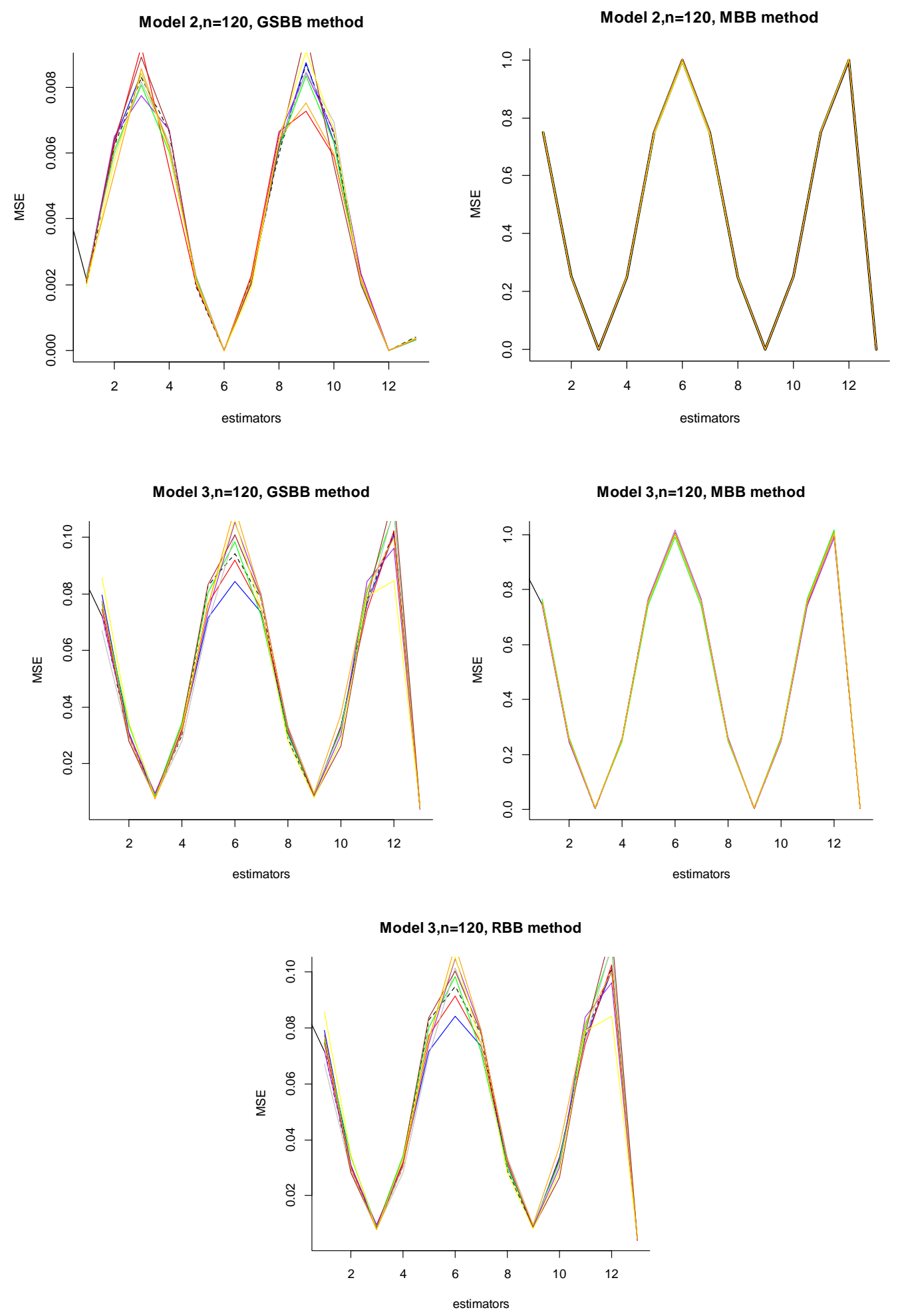

Fig.1 MSE of the estimators of the 12 seasonal means and the overall mean for the models: model 1: $X_{t}=\sin (2 \pi t / T)+e_{t} \cos (2 \pi t / T) ; \quad$ model $\quad 2: \quad X_{t}=\cos (2 \pi t / T)+u_{t} \sin (2 \pi t / T) \quad$ and model $3:$ $X_{t}=e_{t} \cos (2 \pi t / T)+u_{t} \sin (2 \pi t / T)+\cos (2 \pi t / T)$, using GSBB, MBB and RBB methods for all block length .

In the case of MSE for the seasonal means and the overal mean estimates, we can notice that we have similar performance of GSBB and RBB in the three models studied (see figure.1). The values obtained in the case of MBB method are eventually higher than those obtained from the other methods. This indicates the poor perormance of the MBB in this simulation study when calculate MSE. We can notice also that the performance of the three methods is not 
influenced by the choose of the block length. It is recommended to take the block length $b=5$ or $b=12$. In both cases,based on the results obtained from our simulations the performance is similiar. We consider the block length choice $b=5$ in the case of bias of the estimates of seasonal means and the overall mean for all the models studied. The results obtained from three methods are presented in the figure below:

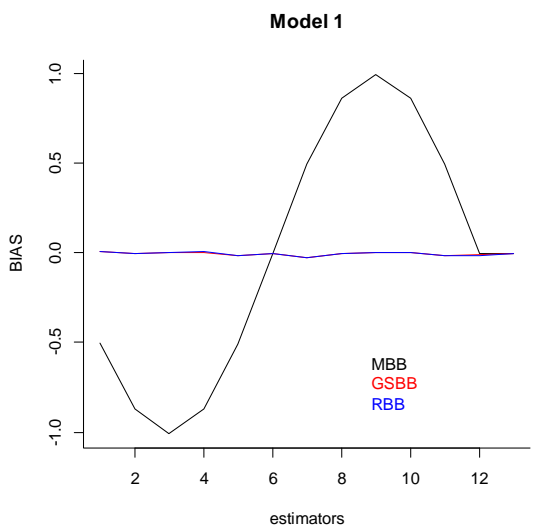

Fig.2 BIAS of the estimators of

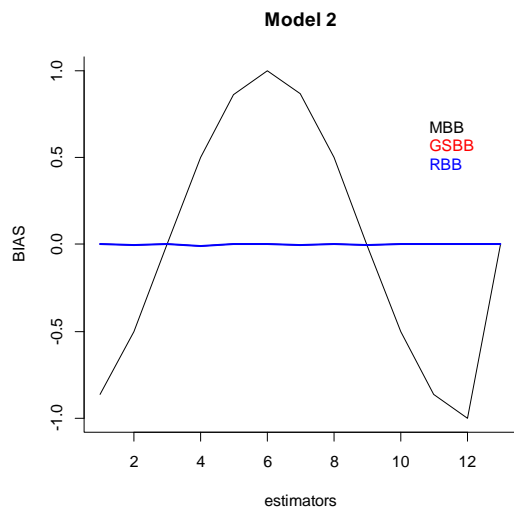

estimators

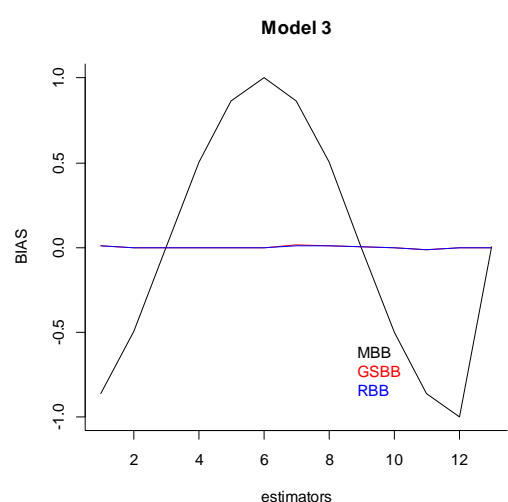
$X_{t}=e_{t} \cos (2 \pi t / T)+u_{t} \sin (2 \pi t / T)+\cos (2 \pi t / T)$, using GSBB, MBB and RBB methods for the optimal block length $\mathrm{b}=5$

The results obtained from the GSBB and RBB are very close to each other (near zero) and they are not distinguishable from each other in the figure 2. However, the performance of MBB is poor even in this case because are obtained values of MSE that are far from zero compared with the results obtained from the other methods: GSBB and RBB. The performance of the GSBB and RBB methods in the three models studied, is better than the performance of the MBB when calculating bias of the estimates of the seasonal means and the overall mean.

\section{CONCLUSIONS}

Based on the results of our simulations, we can conclude that GSBB and RBB have better performance than MBB. The results for each method are not influenced by the block length choice. We can recommend RBB method in the case of similar models because of the reduced time of simulations in comparison of the GSBB method. The poor performance of MBB is caused from the periodic component in the time series studied. GSBB and RBB take in consideration of the periodicity present in the data, and this is the reason of the good performance of these methods in our models of periodically correlated time series.

\section{REFERENCES}

1. Dudek, A.E., Leskow, J., Paparoditis, E. and Politis, D. (2014a). A generalized block bootstrap for seasonal time series. J. Time Ser. Anal., 35 89-114.

2. Efron, B. (1979) Bootstrap methods: Another look at the jackknife, Ann. Statis. 7, 1-26.

3. Hurd, H. L. and Miamee, A. (2007) Periodically Correlated Random Sequences, Spectral Theory and Practice, Whiley \& Sons.

4. Kunsch, H. (1989) The Jackknife and the Bootstrap for general stationary observations, Ann. Stat.17, 1217-1241.

5. Liu, R. Y. and Singh K. (1992) Moving blocks jackknife and bootstrap capture weak dependence. Exploring the Limits of Bootstrap., Wiley New York, 225-248.

6. Margo L. and Ekonomi L. and Milo E. (2015) A Comparison of Block Bootstrap Procedures for Periodic Time Series, FMNS 2015 The Sixth International Conference Of The Faculty Of Mathematics And Natural Sciences, 10-14 june 2015, Blagoevgrad, Bulgaria, Proceeding book, p.92-97, Volume 1.

7. Politis D.N. (2001) Resampling time series with seasonal components, in Frontiers in Data Mining and Bioinformatics. In: Proceedings of the 33rd Symposium on the Interface of Computing Science and Statistics, Orange County, California, pp. 619-621. 